\title{
Periodic Orbits in Polygonal Billiards *
}

\author{
Debabrata Biswast 12 \\ Center for Chaos and Turbulence Studies, Niels Bohr Institute \\ Blegdamsvej 17, Copenhagen Ø, Denmark
}

\begin{abstract}
We review some properties of periodic orbit families in polygonal billiards and discuss in particular a sum rule that they obey. In addition, we provide algorithms to determine periodic orbit families and present numerical results that shed new light on the proliferation law and its variation with the genus of the invariant surface. Finally, we deal with correlations in the length spectrum and find that long orbits display Poisson fluctuations.
\end{abstract}

\section{INTRODUCTION}

Billiards are an interesting and well studied class of hamiltonian systems that display a wide variety of dynamical behaviour depending on the shape of the boundary. The ray equations commonly considered follow from a short wavelength expansion of the Schrödinger equation with Dirichlet (or Neumann) boundary conditions. A particle thus moves freely between collisions at the boundary where it suffers specular reflection. As an example, a square billiard generates regular dynamics that is restricted to a torus in phase space due to

\footnotetext{
*Nonlinearity and Chaos in the Physical Sciences, Pramana (Special Issue)
} 
the existence of two well behaved constants of motion. In contrast, generic trajectories in the enclosure formed by three intersecting discs explore the entire constant energy surface and hence the system is ergodic. Moreover, orbits that are nearby initially move exponentially apart with time and hence the system is said to be metrically chaotic.

Polygonal billiards are a class of systems whose properties are not as well known. The ones accessible to computations are rational angled polygons and they generically belong to the class of systems referred to as pseudo-integrable [3]. They possess two constants of motion like their integrable counterparts 国 but the invariant surface $\Gamma$ is topologically equivalent to a sphere with multiple holes [3] and not a torus. Such systems are characterised by the genus $g$ where $2 g$ is the numbers of cuts required in $\Gamma$ to produce a singly connected region (alternately, $g$ is the number of holes in $\Gamma$ ). As an example, consider the 1-step billiard in Fig. 1. For any trajectory, $p_{x}^{2}$ and $p_{y}^{2}$ are conserved. The invariant surface consists of four sheets (copies) corresponding to the four possible momenta, $\left( \pm p_{x}, \pm p_{y}\right)$ that it can have and the edges of these sheets can be identified such that the resulting surface has the topology of a double torus.

The classical dynamics no longer has the simplicity of an integrable system where a transformation to action and angle co-ordinates enables one to solve the global evolution equations on a torus. On the other hand, the dynamics is non-chaotic with the only interesting feature occurring at the singular vertex with internal angle $3 \pi / 2$. Here, families of parallel rays split and traverse different paths, a fact that limits the extent of periodic orbit families. This is in contrast to integrable billiards (and to the $\pi / 2$ internal angles in Fig. 1) where families of rays do not see the vertex and continue smoothly.

We shall focus here on the periodic orbits of such systems for they form the skeleton on which generic classical motion is built. They are also the central objects of modern semiclassical theories [5] which provide a duality between the quantum spectrum and the classical length and stabilities of periodic orbits. In polygonal billiards, primitive periodic orbits can typically be classified under two categories depending on whether they suffer even or odd number of bounces at the boundary. In both cases however, the linearised flow is 
marginally unstable since the Jacobian matrix, $J_{p}$, connecting the transverse components $\left(u_{\perp}\left(t+T_{p}\right)=J_{p} u_{\perp}(t)\right.$ where $u_{\perp}=\left(q_{\perp}, p_{\perp}\right)^{T}$ and $T_{p}$ is the time period of the orbit) has unit eigenvalues. However, when the number of reflections, $n_{p}$, at the boundary is even, the orbit occurs in a 1-parameter family while it is isolated when $n_{p}$ is odd. This follows from the fact that the left (right) neighbourhood of an orbit becomes the right (left) neighbourhood on reflection so that an initial neighbourhood can never overlap with itself after odd number of reflections [3].

While isolated orbits are important and need to be incorporated in any complete periodic orbit theory, a family of identical orbits has greater weight and such families proliferate faster than isolated orbits [6]. Besides, in a number of cases including the L-shaped billiard of Fig. 1, isolated periodic orbits are altogether absent since the initial and final momenta coincide only if the orbit undergoes even number of reflections. For this reason, we shall consider families of periodic orbits in this article (see Fig. (2) for an example).

Not much is however known about the manner in which they are organised and the few mathematical results that exist [6] concern the asymptotic properties of their proliferation rate. For a sub-class of rational polygons where the vertices and edges lie on an integrable lattice (the so called almost-integrable systems [6]), these asymptotic results are exact. It is known for example that the number of periodic orbit families, $N(l)$, increases quadratically with length, $l$, as $l \rightarrow \infty$. For general rational polygons, rigorous results show that $c_{1} l^{2} \leq$ $N(l) \leq c_{2} l^{2}$ for sufficiently large values of $l$ [6,7] while in case of a regular polygon $P_{n}$ with $n$ sides, it is known that $N(l) \simeq c_{n} l^{2} / A$ [8] where $c_{n}$ is a number theoretic constant and $A$ denotes the area of $P_{n}$. Very little is known however about other aspects such as the sum rules obeyed by periodic orbit families in contrast to the limiting cases of integrable and chaotic behaviour where these have been well studied. Besides, it is desirable to learn about the variation of the proliferation rate as a function of the genus for this should tell us about the transition to chaos in polygonal approximations of chaotic billiards [9, 10].

We shall concern ourselves primarily with a basic sum rule obeyed by periodic orbits arising from the conservation of probability. This leads to the proliferation law, $N(l)=$ 
$\pi b_{0} l^{2} /\langle a(l)\rangle$ where $b_{0}$ is a constant. The quantity $\langle a(l)\rangle$ is the average area occupied by all families of periodic orbits with length less than $l$ and is not a constant unlike integrable billiards. We provide here the some numerical results on how $\langle a(l)\rangle$ changes with the length of the orbits and the genus of the invariant surface. While this does not allow us to make quantitative predictions, the qualitative behaviour sheds new light on the proliferation law for short orbits and its variation with the genus, $g$ of the invariant surface.

Finally, we shall also study correlations in the length spectrum of periodic orbits. The numerical results provided here are the first of its kind for generic pseudo-integrable billiards and corroborate theoretical predictions provided earlier [11].

The organisation of this paper is as follows. In section [1, we provide the basic sum rule and the proliferation law in pseudo-integrable systems. In section [II], we discuss algorithms to determine periodic orbit families in generic situations. This is followed by a numerical demonstration of the results obtained in section $\mathbb{T}$ as well an exploration of the area law. Correlations are discussed in sections $\nabla$ together with numerical results. Our main conclusions are summarised in section $\nabla 1$.

\section{THE BASIC SUM RULE}

The manner in which periodic orbits organise themselves in closed systems is strongly linked to the existence of sum rules arising from conservation laws. For example, the fact that a particle never escapes implies that for chaotic systems [12,13]

$$
\left\langle\sum_{p} \sum_{r=1}^{\infty} \frac{T_{p} \delta\left(t-r T_{p}\right)}{\left|\operatorname{det}\left(\mathbf{1}-\mathbf{J}_{p}{ }^{r}\right)\right|}\right\rangle=1
$$

where the summation over p refers to all primitive periodic orbits, $T_{p}$ is time period, $\mathbf{J}_{p}$ is the stability matrix evaluated on the orbit and the symbol $\langle$.$\rangle denotes the average value of the$ expression on the left. Since the periodic orbits are unstable and isolated, $\left|\operatorname{det}\left(\mathbf{1}-\mathbf{J}_{p}{ }^{r}\right)\right| \simeq$ $e^{\lambda_{p} r T_{p}}$, where $\lambda_{p}$ is the Lyapunov exponent of the orbit. The exponential proliferation of orbits is thus implicit in eq. (四). 
A transparent derivation of Eq. (11) follows from the classical evolution operator

$$
L^{t} \circ \phi(\mathbf{x})=\int \mathbf{d y} \delta\left(\mathbf{x}-\mathbf{f}^{t}(\mathbf{y})\right) \phi(\mathbf{y})
$$

where $L^{t}$ governs the evolution of densities $\phi(\mathbf{x}), \mathbf{x}=(\mathbf{q}, \mathbf{p})$ and $\mathbf{f}^{t}$ refers to the flow in the full phase space. We denote by $\Lambda_{n}(t)$ the eigenvalue corresponding to an eigenfunction $\phi_{n}(\mathbf{x})$ such that $L^{t} \circ \phi_{n}(\mathbf{x})=\Lambda_{n}(t) \phi_{n}(\mathbf{x})$. The semi-group property, $L^{t_{1}} \circ L^{t_{2}}=L^{t_{1}+t_{2}}$, for continuous time implies that the eigenvalues $\left\{\Lambda_{n}(t)\right\}$ are of the form $\left\{e^{\lambda_{n} t}\right\}$. Further, for hamiltonian flows, Eq. (22) implies that there exists a unit eigenvalue corresponding to a uniform density so that $\lambda_{0}=0$. For strongly hyperbolic systems, $\lambda_{n}=-\alpha_{n}+i \beta_{n}, n>1$ with a negative real part implying that

$$
\operatorname{Tr} L^{t}=1+\sum_{n} \exp \left\{-\alpha_{n} t+i \beta_{n} t\right\}
$$

Eq. (11) is thus a restatement of Eq. (3) with the trace expressed in terms of periodic orbit stabilities and time periods.

For polygonal billiards, appropriate modifications are necessary to take account of the fact that the flow is restricted to an invariant surface that is two dimensional. Further, since classical considerations do not always yield the spectrum $\left\{\lambda_{n}(t)\right\}$, we shall take resort to the semiclassical trace formula which involves periodic orbit sums similar to the kind that we shall encounter in the classical case.

Before considering the more general case of pseudo-integrable billiards, we first introduce the appropriate classical evolution operator for integrable systems. This is easily defined as

$$
L^{t} \circ \phi\left(\theta_{1}, \theta_{2}\right)=\int d \theta_{1}^{\prime} d \theta_{2}^{\prime} \delta\left(\theta_{1}-\theta_{1}^{\prime t}\right) \delta\left(\theta_{2}-\theta_{2}^{\prime t}\right) \phi\left(\theta_{1}^{\prime}, \theta_{2}^{\prime}\right)
$$

where $\theta_{1}$ and $\theta_{2}$ are the angular coordinates on the torus and evolve in time as $\theta_{i}^{t}=$ $\omega_{i}\left(I_{1}, I_{2}\right) t+\theta_{i}$ with $\omega_{i}=\partial H\left(I_{1}, I_{2}\right) / \partial I_{i}$ and $I_{i}=\frac{1}{2 \pi} \oint_{\Gamma_{i}}$ p.dq. Here $\Gamma_{i}, i=1,2$ refer to the two irreducible circuits on the torus and $\mathbf{p}$ is the momentum conjugate to the coordinate $\mathbf{q}$. 
It is easy to see that the eigenfunctions $\left\{\phi_{n}\left(\theta_{1}, \theta_{2}\right)\right\}$ are such that $\phi_{n}\left(\theta_{1}^{t}, \theta_{2}^{t}\right)=$ $\Lambda_{n}(t) \phi_{n}\left(\theta_{1}, \theta_{2}\right)$ where $\Lambda_{n}(t)=e^{i \alpha_{n} t}$. On demanding that $\phi_{n}\left(\theta_{1}, \theta_{2}\right)$ be a single valued function of $\left(\theta_{1}, \theta_{2}\right)$, it follows that $\phi_{\mathbf{n}}\left(\theta_{1}, \theta_{2}\right)=e^{i\left(n_{1} \theta_{1}+n_{2} \theta_{2}\right)}$ where $\mathbf{n}=\left(n_{1}, n_{2}\right)$ is a point on the integer lattice. Thus the eigenvalue, $\Lambda_{\mathbf{n}}(t)=\exp \left\{i t\left(n_{1} \omega_{1}+n_{2} \omega_{2}\right)\right\}$.

To illustrate this, we consider a rectangular billiard where the hamiltonian expressed in terms of the actions, $I_{1}, I_{2}$ is $H\left(I_{1}, I_{2}\right)=\pi^{2}\left(I_{1}^{2} / L_{1}^{2}+I_{2}^{2} / L_{2}^{2}\right)$ where $L_{1}, L_{2}$ are the lengths of the two sides. With $I_{1}=\sqrt{E} L_{1} \cos (\varphi) / \pi$ and $I_{2}=\sqrt{E} L_{2} \sin (\varphi) / \pi$, it is easy to see that at a given energy, $E$, each torus is parametrised by a particular value of $\varphi$. Thus

$$
\Lambda_{\mathbf{n}}(t)=e^{i 2 \pi t \sqrt{E}\left(n_{1} \cos (\varphi) / L_{1}+n_{2} \sin (\varphi) / L_{2}\right)}
$$

and the spectrum is continuous. The trace thus involves an integration over $\varphi$ as well as a sum over all $n_{1}, n_{2}$ :

$$
\operatorname{Tr} L^{t}=\sum_{\mathbf{n}} \int_{-\pi-\mu_{n}}^{\pi-\mu_{n}} d \varphi e^{i l \sqrt{E_{\mathbf{n}}} \sin \left(\varphi+\mu_{\mathbf{n}}\right)}=2 \pi \sum_{\mathbf{n}} J_{0}\left(\sqrt{E_{\mathbf{n}}}\right)
$$

where $J_{0}$ is a Bessel function, $l=2 t \sqrt{E}, \tan \left(\mu_{\mathbf{n}}\right)=n_{1} L_{2} /\left(n_{2} L_{1}\right)$ and $E_{n}=\pi^{2}\left(n_{1}^{2} / L_{1}^{2}+\right.$ $\left.n_{2}^{2} / L_{2}^{2}\right)$. On separating out $\mathbf{n}=(0,0)$ from the rest and restricting the summation to the first quadrant of the integer lattice, it follows that

$$
\operatorname{Tr} L^{t}=2 \pi+2 \pi N \sum_{n} J_{0}\left(\sqrt{E_{n}} l\right) .
$$

where $N=4$. Note that the first term on the right merely states the fact that there exists a unit eigenvalue on every torus labelled by $\varphi$. Also, though we have not invoked semiclassics at any stage, the spectrum $\left\{E_{n}\right\}$ corresponds to the Neumann spectrum of the billiard considered. We shall subsequently show that this is true in general and for now it remains to express the trace of the evolution operator in terms of periodic orbits.

The trace of $L^{t}$ in the integrable case can be expressed as

$$
\operatorname{Tr} L^{t}=\int d \varphi \int d \theta_{1} d \theta_{2} \delta\left(\theta_{1}-\theta_{1}^{t}\right) \delta\left(\theta_{2}-\theta_{2}^{t}\right)
$$

It follows immediately that the only orbits that contribute are the ones that are periodic. For a rectangular billiard, the integrals can be evaluated quite easily and yields 


$$
\operatorname{Tr} L^{t}=4 \sum_{N_{1}} \sum_{N_{2}} \frac{4 L_{1} L_{2}}{l_{N_{1}, N_{2}}} \delta\left(l-l_{N_{1}, N_{2}}\right)
$$

where $\left\{N_{1}, N_{2}\right\}$ are the winding numbers on the torus and label periodic orbits of length $l_{N_{1}, N_{2}}$. Using Eqns. (7) and (9), it follows that

$$
4 \sum_{N_{1}} \sum_{N_{2}} \frac{4 L_{1} L_{2}}{l_{N_{1}, N_{2}}} \delta\left(l-l_{N_{1}, N_{2}}\right)=2 \pi+2 \pi N \sum_{n} J_{0}\left(\sqrt{E_{n}} l\right)
$$

Thus, the dominant non-oscillatory contribution to the trace that survives averaging is $2 \pi$ and this gives rise to the analogue of Eq. (11)

$$
4\left\langle\sum_{N_{1}} \sum_{N_{2}} \frac{4 L_{1} L_{2}}{l_{N_{1}, N_{2}}} \delta\left(l-l_{N_{1}, N_{2}}\right)\right\rangle=2 \pi
$$

The proliferation rate for the rectangular billiard thus follows from these considerations.

These ideas can be generalised for polygonal billiards that are pseudo-integrable even though the structure of the invariant surface no longer allows one to use action and angle variables.

For both integrable and pseudo-integrable polygonal billiards, the dynamics in phase space can be viewed in a singly connected region by executing $2 g$ cuts in the invariant surface and identifying edges appropriately. At a given energy, the motion is parametrised by the angle, $\varphi$, that a trajectory makes with respect to one of the edges. As a trivial example, consider the rectangular billiard. The singly connected region is a larger rectangle consisting of four copies corresponding to the four directions that a trajectory can have and these can be glued appropriately to form a torus [14]. As a non-trivial example, consider the L-shaped billiard of Fig. (1) which is pseudo-integrable with its invariant surface having, $g=2$. Alternately, the surface can be represented by a singly connected region in the plane (see Fig. (3)) and consists of four copies corresponding to the four possible directions an orbit can have and these are glued appropriately. A trajectory in phase space thus consists of parallel segments at an angle $\varphi$ measured for example with respect to one of the sides. It will be useful to note at this point that the same trajectory can also be represented by parallel segments at angles $\pi-\varphi, \pi+\varphi$ and $2 \pi-\varphi$. In general, the number of directions 
for representing a trajectory equals the number of copies, $N$, that constitute the invariant surface.

The classical propagator on an invariant surface parametrised by $\varphi$ is thus

$$
L^{t}(\varphi) \circ \phi(\mathbf{q})=\int d \mathbf{q}^{\prime} \delta\left(\mathbf{q}-\mathbf{q}^{\prime t}(\varphi)\right) \phi\left(\mathbf{q}^{\prime}\right)
$$

where $\mathbf{q}$ refers to the position in the singly connected region and $\mathbf{q}^{\prime t}(\varphi)$ is the time evolution parametrised by $\varphi$ as described above.

The trace of $L^{t}(\varphi)$ takes into account all possible invariant surfaces that exist and hence involves an additional integration over $\varphi$. Thus

$$
\operatorname{Tr} L^{t}=\int d \varphi \int d \mathbf{q} \delta\left(\mathbf{q}-\mathbf{q}^{t}(\varphi)\right)
$$

Clearly the only orbits that contribute are the ones that are periodic. Further, the $\mathbf{q}$ integrations are simpler if we transform to a local coordinate system with one component parallel to the trajectory and the other perpendicular. Thus $\delta_{\|}\left(q_{\|}-q_{\|}^{t}\right)=\frac{1}{v} \delta\left(t-r T_{p}\right)$ where $v$ is the velocity, $T_{p}$ is the period of the orbit and $r$ is the repetition number. Similarly, for an orbit of period $T_{p}$ parametrised by the angle $\varphi_{p}, \delta_{\perp}\left(q_{\perp}-q_{\perp}^{t}\right)=\delta\left(\varphi-\varphi_{p}\right) /\left|\partial q_{\perp} / \partial \varphi\right|_{\varphi=\varphi_{p}}$ where $\left|\partial q_{\perp} / \partial \varphi\right|_{\varphi=\varphi_{p}}=r l_{p}$ for marginally unstable billiards. Putting these results together and noting that each periodic orbit occurs in general at $N$ different values of $\varphi$, we finally have

$$
\operatorname{Tr} L^{t}=\sum_{n} \Lambda_{n}(t)=N \sum_{p} \sum_{r=1}^{\infty} \frac{a_{p}}{r l_{p}} \delta\left(l-r l_{p}\right)
$$

where $l=t v$ and the summation over $p$ refers to all primitive periodic orbit families with length $l_{p}$ and occupying an area $a_{p}$. Note that Eq. (9) is a special case of Eq. (14) which holds for both integrable and non-integrable polygonal billiards.

It is possible to re-express the periodic orbit sum in Eq. (14) starting with the appropriate quantum trace formula [15]

$$
\sum_{n} \delta\left(E-E_{n}\right)=d_{a v}(E)+\frac{1}{\sqrt{8 \pi^{3}}} \sum_{p} \sum_{r=1}^{\infty} \frac{a_{p}}{\sqrt{k r l_{p}}} \cos \left(k r l_{p}-\frac{\pi}{4}-\pi r n_{p}\right)
$$


Here $d_{a v}(E)$ refers to the average density of quantal eigenstates, $k=\sqrt{E}, l_{p}$ is the length of a primitive periodic orbit family. The phase $\pi r n_{p}$ is set to zero while considering the Neumann spectrum while in the Dirichlet case, $n_{p}$ equals the number of bounces that the primitive orbit suffers at the boundary. For convenience, we have chosen $\hbar=1$ and the mass $m=1 / 2$. Starting with the function

$$
g(l)=\sum_{n} f\left(\sqrt{E_{n}} l\right) e^{-\beta E_{n}}=\int_{\epsilon}^{\infty} d E f(\sqrt{E} l) e^{-\beta E} \sum_{n} \delta\left(E-E_{n}\right)
$$

where $f(x)=\sqrt{\frac{2}{\pi x}} \cos (x-\pi / 4)$ and $0<\epsilon<E_{0}$, it is possible to show using Eq. (15) that for polygonal billiards 17

$$
\sum_{p} \sum_{r=1}^{\infty} \frac{a_{p}}{r l_{p}} \delta\left(l-r l_{p}\right)=2 \pi b_{0}+2 \pi \sum_{n} f\left(\sqrt{E_{n}} l\right)
$$

for $\beta \rightarrow 0^{+}$. In the above,

$$
b_{0}=\sum_{p} \sum_{r} \frac{a_{p}(-1)^{r n_{p}}}{4 \pi} \int_{0}^{\epsilon} d E f(\sqrt{E} l) f\left(\sqrt{E} r l_{p}\right)
$$

and is a constant [17, 18]. It follows from Eqns. (17) and (14) that

$$
\operatorname{Tr} L^{t}=\sum_{p} \sum_{r=1}^{\infty} \frac{N a_{p}}{r l_{p}} \delta\left(l-r l_{p}\right)=2 \pi N b_{0}+2 \pi N \sum_{n} f\left(\sqrt{E_{n}} l\right) .
$$

where $\left\{E_{n}\right\}$ are the Neumann eigenvalues of the system. As in case of the rectangular billiard, the oscillatory contributions wash out on averaging so that

$$
\left\langle\sum_{p} \sum_{r=1}^{\infty} \frac{a_{p}}{r l_{p}} \delta\left(l-r l_{p}\right)\right\rangle=2 \pi b_{0}
$$

This is the central result of this section and forms the basic sum rule obeyed by periodic orbit families.

A few remarks about this derivation and the magnitude of $b_{0}$ are however in order. Eq. (14) is exact for the L-shaped billiard and all other boundaries which preclude the existence of isolated periodic orbits. However, even for these shapes, the semiclassical trace formula is only an approximation to the exact density whenever the billiard in question is pseudo-integrable. In this sense, the sum rule in Eq. (20) is not expected to be exact. 
However, we believe the existence of higher order corrections (such as diffraction) affects the magnitude of the constant $b_{0}$ while preserving the constant nature of the periodic orbit sum on the left.

In the integrable case it is easy to show from other considerations that $b_{0}=1 / N$ where $N$ is the number of sheets that constitute the invariant surface [17]. This also follows from Eqns. (10) and (19) since $b_{0} N=1$. For pseudo-integrable billiards, each invariant surface parametrised by $\varphi$ has an eigenvalue, $\Lambda_{0}(\varphi)=1$. Thus the non-oscillatory part of the trace should equal $\int \Lambda_{0}(\varphi) d \varphi$ and to a first approximation this yields $2 \pi$ implying that $b_{0}=1 / N$. However each singular vertex connects two distinct points at any angle $\varphi$ and hence the integration over $\varphi$ is non-trivial. We can therefore state that $b_{0}$ is approximately $1 / N$ in the pseudo-integrable case while this is exact in the integrable case. We shall show numerically that the magnitude of deviations (from $1 / N$ ) in the pseudo-integrable case depends on the existence of periodic orbit pairs at the singular vertex. First however, we briefly describe the algorithms used to determine periodic orbit families.

\section{ALGORITHMS FOR DETERMINING PERIODIC ORBITS}

Periodic orbits in polygonal billiards are generally hard to classify. Unlike integrable billiards, they cannot be described by two integers which count the number of windings around the two irreducible circuits on the torus though in exceptional cases this can indeed be done. However, since the invariant surface has a well-defined genus, it is expected that a set of integers $\mathbf{N}=\left\{N_{1}, N_{2}, \ldots, N_{2 g}\right\}$ obeying the relationship

$$
\omega_{i}=\frac{2 \pi N_{i}}{T_{\mathbf{N}}}
$$

can be used to label periodic orbits. Here $\omega_{i}$ refers to the frequency corresponding to each irreducible circuit $\Gamma_{i}$ and depends on the energy, $\mathrm{E}$ and the angle $\varphi$ that labels each invariant surface. Note however that not all points on this multi-dimensional integer lattice are allowed since there are constraints and this method of labelling orbits becomes cumbersome for 
surfaces of higher genus. Nevertheless, we illustrate the idea here for the L-shaped billiard of Fig. (1).

Let the length of the two bouncing ball orbits in the X-direction be $L_{1}$ and $L_{2}$ respectively and their lengths in the Y-direction be $L_{3}$ and $L_{4}$. These define the irreducible circuits for the L-shaped billiard. Thus :

$$
\begin{aligned}
& \frac{v \cos (\varphi)}{2 L_{1}}=\frac{2 \pi N_{1}}{T_{\mathbf{N}}} \\
& \frac{v \cos (\varphi)}{2 L_{2}}=\frac{2 \pi N_{2}}{T_{\mathbf{N}}} \\
& \frac{v \sin (\varphi)}{2 L_{3}}=\frac{2 \pi N_{3}}{T_{\mathbf{N}}} \\
& \frac{v \sin (\varphi)}{2 L_{4}}=\frac{2 \pi N_{4}}{T_{\mathbf{N}}}
\end{aligned}
$$

This implies that the angle $\varphi$ at which a periodic orbit can exist is such that

$$
\tan (\varphi)=\frac{N_{3} L_{3}+N_{4} L_{4}}{N_{1} L_{1}+N_{2} L_{2}}
$$

Eqns. (23) and (22) merely express the fact that any periodic orbit should have integer number of windings around the irreducible circuits. Thus, the total displacement along the X-direction should be $2\left(N_{1} L_{1}+N_{2} L_{2}\right)$ while the total displacement in the Y-direction should be $2\left(N_{3} L_{3}+N_{4} L_{4}\right)$ where $N_{i}$ are integers. As mentioned before however, not all realizations of $\left\{N_{i}\right\}$ correspond to real periodic orbits and the final step consists in checking numerically whether a periodic orbit at the angle $\varphi$ (given by Eq. (23)) exists. Note that one member of each family necessarily resides at one of the singular vertices and hence it is sufficient to verify the existence of this orbit.

This method works equally well for other billiards with steps (see Fig. (1)) and the number of integers necessary to describe orbits increases with the number of steps. An alternate method which exploits the fact that periodic orbits occur in families is often useful when the irreducible circuits are not obvious and this is described below.

Note that a non-periodic orbit originating from the same point $\mathbf{q}$ (e.g. the singular vertex) as a periodic orbit but with a momentum slightly different (from the periodic orbit) 
suffers a net transverse deviation $q_{\perp}=(-1)^{n_{\varphi}} \sin \left(\varphi-\varphi_{p}\right) l_{\varphi}$. Here $l_{\varphi}$ is the distance traversed by a non-periodic orbit at an angle $\varphi$ after $n_{\varphi}$ reflections from the boundary and $\varphi_{p}$ is the angle at which a periodic orbit exists. This provides a correction to the initial angle and a few iterations are normally sufficient to converge on a periodic orbit with good accuracy. In order to obtain all periodic orbits, it is necessary to shoot trajectories from every singular vertex since one member of each family resides at one of these vertices.

Apart from the length of a periodic orbit, it is also important to compute the area occupied by the family. This can be achieved by shooting a single periodic trajectory which resides at a singular vertex and by noting that this orbit lies on the edge of a family. Thus if the rest of the family lies on the left neighbourhood initially, it is necessary to determine the perpendicular distance from a singular vertex to this trajectory every time the initial neighbourhood lies towards this singular vertex. The shortest of these perpendicular distances gives the transverse extent of the family and the area can thus be computed.

These algorithms have been used to generate the lengths $\left\{l_{p}\right\}$ and areas $\left\{a_{p}\right\}$ of primitive orbits in the L-shaped billiard as well as the two and three step billiards. We present our numerical results in the following sections.

\section{NUMERICAL RESULTS : THE SUM RULE AND AREA LAW}

We present here our numerical results on the 1-step (L-shaped), 2-step and 3-step billiards. Their invariant surfaces have genus 2, 3 and 4 respectively and the quantities we shall study are the sum rule derived in section $\mathbb{\amalg}$ and the variation of $\langle a(l)\rangle$ with $l$ and the genus, $g$ of the invariant surface.

The sum rule we wish to study can be re-expressed as

$$
S(l)=\frac{1}{2 \pi} \sum_{p} \sum_{r} \frac{a_{p}}{r l_{p} \leq l} \underset{r}{p} \simeq b_{0} l
$$

and this is plotted in Fig. (4) for four different 1-step (L-shaped) billiards. Notice first that in each case the behaviour is linear as predicted by Eq. (24). Besides, in three of the four 
cases, the slopes are quite close $\left(b_{0} \simeq 0.27\right)$ and these correspond to non-degenerate L-shaped billiards with sides that are unequal and irrationally related. The one with a substantially larger slope $\left(b_{0} \simeq 0.32\right)$ is a degenerate case of a square with a quarter removed and for this system there exist a substantial number of periodic orbit pairs at the same angle on the two adjacent edges at the singular vertex. The differences between the degenerate and non-degenerate case also persists in other quantities as we shall shortly see.

We next plot $S(l)$ for non-degenerate examples of a 1,2 and 3-step billiards in Fig. (5). The number of orbits considered are far less due to increased computational effort though the linear behaviour is obvious in all three cases. The slopes are again close to 0.25 and vary from $b_{0} \simeq 0.24$ to $b_{0} \simeq 0.28$

Thus, periodic orbits obey a basic sum rule given by Eq. (24) where $b_{0}$ is a constant. Further, the magnitude of $b_{0}$ is close to 0.25 in all cases and the deviations from this value are larger in the degenerate case where periodic orbit pairs exist at the singular vertex.

The sum rule yields the proliferation rate of periodic orbits

$$
N(l)=\frac{\pi b_{0} l^{2}}{\langle a(l)\rangle}
$$

where $\langle a(l)\rangle$ is the average area occupied by periodic orbit families with length less than $l$. In case of integrable polygons $\langle a(l)\rangle$ is a constant and equals $A N$ where $A$ is the area of the billiard while $b_{0}=1 / N$. For pseudo-integrable cases, the areas $a_{p}$ occupied by individual periodic orbit families generically occupy a wide spectrum bounded above by $N A$. As the length of a family increases, encounters with singular vertices are more frequent so that the transverse extent of family decreases. Quantitative predictions about the behaviour of $\langle a(l)\rangle$ are however difficult and we provide here some numerical results.

Fig. (6) shows a plot of $\langle a(l)\rangle / N A$ for three different L-shaped billiards one of which is the degenerate case presented in Fig. (4). The average area increases initially before saturating for large $l$ in all cases. The normalised saturation value seems to be independent of the ratio of the sides as long as they are irrationally related (we have observed this for other cases not presented here) but is very different for the degenerate example of a square 
with a quarter removed.

A comparison of non-degenerate 1,2 and 3-step billiards is shown in Fig. (7). Recall that the invariant surfaces for these have genus respectively equal to 2,3 and 4 . The saturation observed for the 1-step (L-shaped) case seems to be a common feature and interestingly the normalised average saturation value decreases. This is however expected since the number of singular vertices increases with the genus thereby reducing the average transverse extent of orbit families at any given length.

These observations allow us to conclude that for short lengths, the proliferation law is sub-quadratic. Asymptotically however, $N(l) \sim l^{2}$ as in integrable billiards. Further, the asymptotic proliferation rate for billiards with the same area $A$ increases with the genus due to a decrease in the asymptotic normalised saturation value of $\langle a(l)\rangle$.

These numerical results provide a qualitative picture of the area law and show that periodic orbits in polygonal billiards are organised such that they obey a sum rule. Quantitative predictions would require a more extensive numerical study such that empirical laws for the saturation value and its variation with the genus can be arrived at.

\section{CORRELATIONS IN THE LENGTH SPECTRUM}

Our numerical explorations so far have been focussed on the average properties of the length spectrum. We shall now attempt to understand the nature of the fluctuations and characterise their statistical properties.

Fluctuations in the length spectrum are generally difficult to study from purely classical considerations. There are notable exceptions however. Fluctuations in the integrable case can be studied using the Poisson summation formula since the lengths of orbits are expressed in terms of integers $\left\{N_{1}, N_{2}\right\}$ [19]. The other extreme is the motion on surfaces of constant negative curvature where the Selberg trace formula provides an exact dual relationship between the classical lengths and the eigenvalues of the Laplace-Beltrami operator [20]. For other systems, a possible way of studying fluctuations in the length spectrum lies 
in inverting the semiclassical quantum trace formula. For pseudo-integrable billiards, this has been achieved in section $\mathbb{I}$ and the integrable density of lengths, $N(l)$ can be expressed as

$$
N(l)=\frac{\pi b_{0} l^{2}}{\langle a(l)\rangle}+\frac{2 \pi}{\langle a(l)\rangle} \sum_{n} \int_{0}^{l} d l^{\prime} l^{\prime} f\left(\sqrt{E_{n}} l^{\prime}\right)
$$

Statistical properties of the fluctuations can thus be studied using techniques introduced for the fluctuations in the quantum energy spectrum [21,11].

The correlations commonly studied are the nearest neighbour spacings distribution, $P(s)$ and a two-point correlation referred to as the spectral rigidity, $\Delta_{3}(L)$. The rigidity measures the average mean square deviation of the staircase function $N(l)$ from the best fitting straight line over $L$ mean level spacings. For a normalised (unit mean spacing) Poisson spectrum, $P(s)=e^{-s}$ while $\Delta_{3}(L)=L / 15$. These features are commonly observed in the quantum energy spectrum of integrable systems as well as in their length spectrum. For chaotic billiards, the quantum energy spectrum generically produces non-Poisson statistics while the length spectrum correlations are Poisson for long orbits at least over short ranges [22]. There are however deviations that can be observed in $\Delta_{3}(L)$ for short orbits or over longer ranges in the spectrum [22]. With this background, we now present our results for pseudointegrable billiards.

Figs. (8) and (9) show plots of $P(s)$ and $\Delta_{3}(L)$ for a non-degenerate L-shaped billiard. A total of 3000 lengths have been considered after excluding the shortest 3000 orbits. The correlations are clearly Poissonian as in case of integrable or chaotic billiards.

For the 3-step billiard where fewer lengths are available (about 1250), we have carried out a similar study and the results are shown in Figs. (10) and (11). Deviations from the Possion behaviour can now be seen especially in the spectral rigidity. By considering shorter lengths in the L-shaped billiard, similar deviations were observed.

The statistical properties of fluctuations in the length spectrum of pseudo-integrable systems are thus similar to those of chaotic billiards. For long orbits the correlations are Poisson while deviations exist for shorter orbits. 


\section{DISCUSSIONS AND CONCLUSIONS}

Quantum billiards are experimentally realizable in the form of microwave cavities since the wave equations are identical [23,24]. They are also interesting in their own right and have proved to be the testing grounds for several ideas in the field of Quantum Chaos. Of all possible shapes, polygonal billiards have perhaps been the least understood largely because very little was known about the organisation of periodic orbits. The results presented here have however been used recently to obtain convergent semiclassical eigenvalues with arbitrary non-periodic trajectories [25] as well to demonstrate that periodic orbits provide excellent estimates of two-point correlations in the quantum energy spectrum [18. Though it is beyond the scope of this article to review these recent developments on quantum polygonal billiards, we refer the interested reader to these articles and the references contained therein.

In the previous sections we have explored the organisation of periodic orbits in rational polygonal billiards that are pseudo-integrable. Our main conclusions are as follows :

- Orbit families obey the sum rule $\left\langle\sum_{p} \sum_{r=1}^{\infty} a_{p} \delta\left(l-r l_{p}\right) /\left(r l_{p}\right)\right\rangle=2 \pi b_{0}$ thereby giving rise to the proliferation law $N(l)=\pi b_{0} l^{2} /\langle a(l)\rangle$ for all rational polygons.

- The quantity $b_{0}$ is approximately $1 / N$ in generic generic rational billiards and deviations from this value are observed to be significant in degenerate situations.

- $\langle a(l)\rangle$ increases initially before saturating to a value much smaller than the maximum allowed area $N A$. The asymptotic proliferation law is thus quadratic even for systems that are not almost-integrable and the density of periodic orbits lengths is far greater than an equivalent integrable systems having the same area.

- The normalised average area $\langle a(l)\rangle / N A$ decreases with the genus of the billiard while $b_{0}$ is approximately 0.25 . Periodic orbits thus proliferate faster with an increase in genus.

- The statistical properties of the fluctuations in the length spectrum are Poisson when the orbits considered are long. 


\section{ACKNOWLEDGEMENTS}

Part of the work reviewed here was published earlier in collaboration with Sudeshna Sinha and it a pleasure to acknowledge the numerous discussions we had on this subject. I have also benifitted directly or indirectly from interesting discussions with Predrag Cvitanović, Gregor Tanner and Bertrand Gerogeot. 


\section{REFERENCES}

[1] email : biswas@kaos.nbi.dk

[2] On leave from the Theoretical Physics Division, Bhabha Atomic Research Centre, Bombay 400 085, India.

[3] P.J.Richens and M.V.Berry, Physica D2, 495(1981).

[4] The few examples of integrable polygons are the rectangle and triangles with internal angles $(\pi / 3, \pi / 3, \pi / 3)),(\pi / 3, \pi / 6, \pi / 2)$ and $(\pi / 4, \pi / 4, \pi / 2)$.

[5] M.C.Gutzwiller, Chaos in Classical and Quantum Mechanics, Springer, New York, 1990.

[6] E.Gutkin, Physica D19,311(1986); J. Stat. Phys 83, 7 (1996).

[7] H.Masur, Ergod. Th. and Dyn. Sys. 10, 151(1990); MSRI,215(1988).

[8] W.A.Veech, Geo. and Func. Anal. 2, 341(1992).

[9] J.Vega, T.Uzer and J.Ford, Phys. Rev. E48, 3414 (1993).

[10] T.Cheon and T.D.Cohen, Phys. Rev. Lett. 62,2769(19891).

[11] D.Biswas and S.Sinha, Phys. Lett. A, 173, 392 (1993).

[12] P.Cvitanović and B.Eckhardt, J. Phys. A. 24, L237 (1991).

[13] J.H.Hannay and A.M.Ozorio de Almeida, J.Phys. A17,3429(1984).

[14] J.B.Keller and S.I.Rubinow, Ann. Phys. (N.Y.) 9, 24(1960).

[15] The respective contributions of isolated and diffractive periodic orbits are $O\left(k^{-1}\right)$ and $O\left(k^{-1-\nu / 2}\right)$ where $\nu$ is the total number of (singular) vertex connections in a diffractive periodic orbit [16].

[16] N.D.Whelan, Phys. Rev. E 51,3778(1995); N. Pavloff and C. Schmit, Phys. Rev. Lett. $75,61(1995)$. 
[17] D.Biswas and S.Sinha, Phys. Rev. Lett. 70, 916(1993)

[18] D.Biswas, Phys. Rev. E, 54, R1044(1996).

[19] D.Biswas, Pramana - Journal of Physics, 42, 447 (1994).

[20] A.Selberg, J. Indian Math. Soc. 20, 47 (1956); N.Balazs and A.Voros, Phys. Rep. 143, 109 (1986).

[21] M.V.Berry, Proc. Roy. Soc. Lond. Ser. A400, 229(1985).

[22] D.Biswas, Phys. Rev. Lett. 71, 2714 (1993).

[23] H.-J. Stockmann and J. Stein, Phys. Rev. Lett. 64, 2867 (1990).

[24] S.Sridhar, Phys. Rev. Lett. 67, 785 (1991).

[25] D.Biswas, Quasi-Classical Quantization, to be published. 


\section{FIGURES}

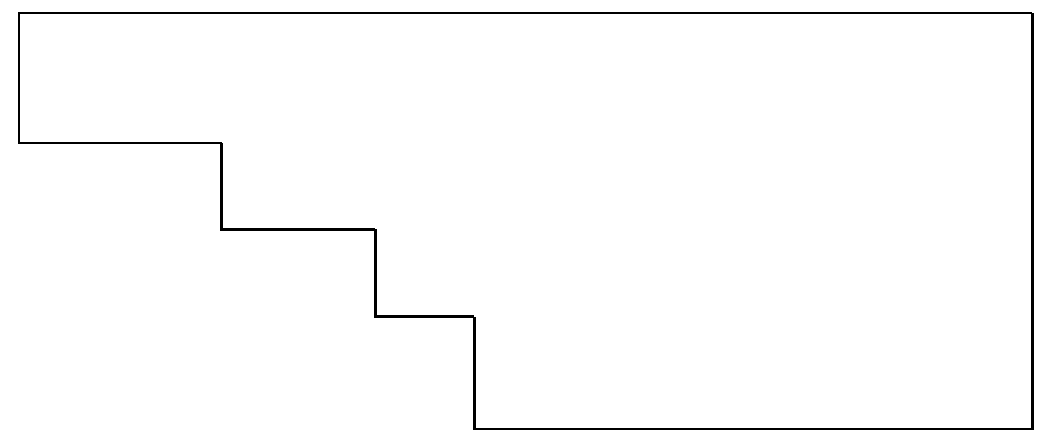

$Y$
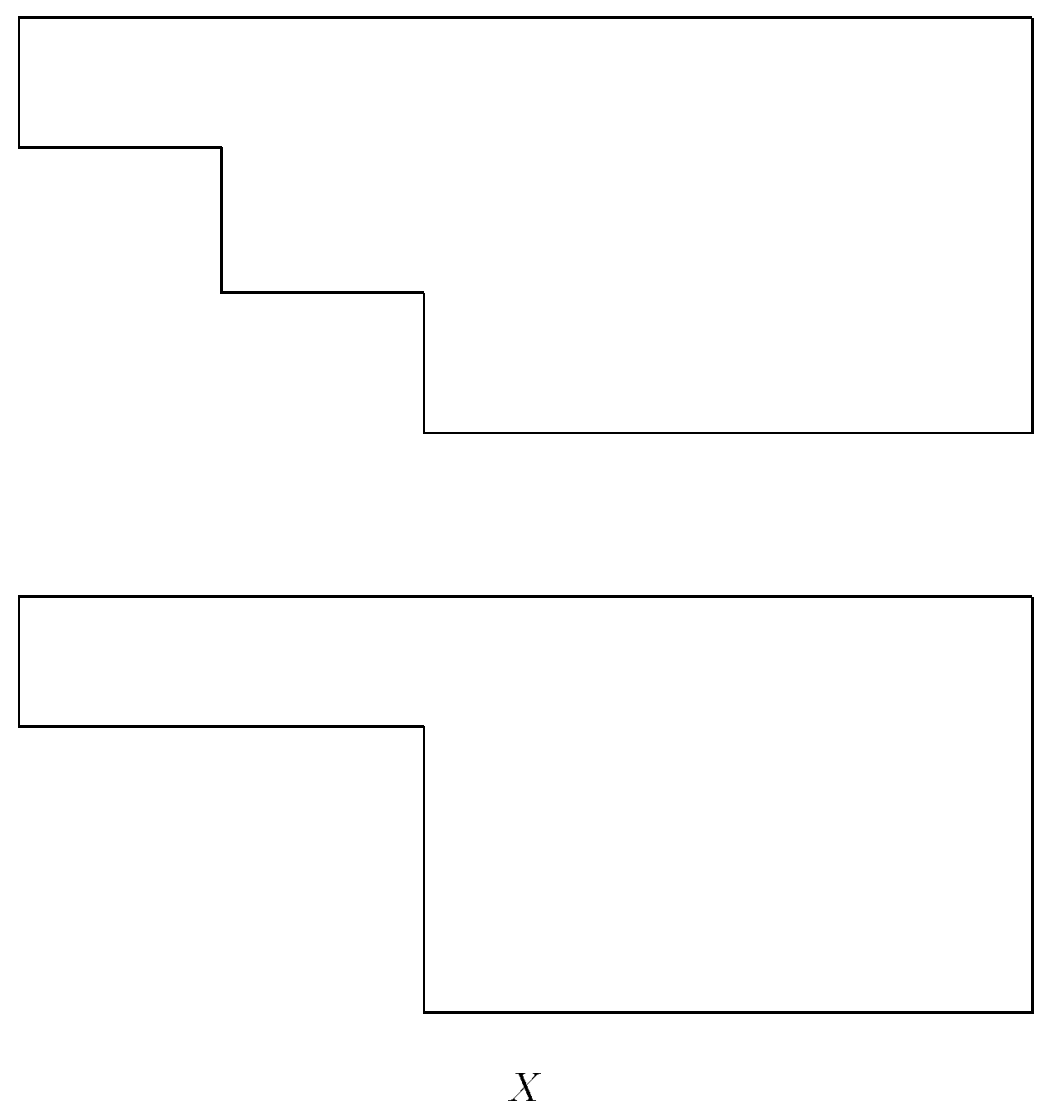

FIG. 1. The 1,2 and 3 -step billiards are pseudo-integrable due to the $3 \pi / 2$ internal angles. The 1-step billiard (bottom) is also referred to as the L-shaped billiard in the text 


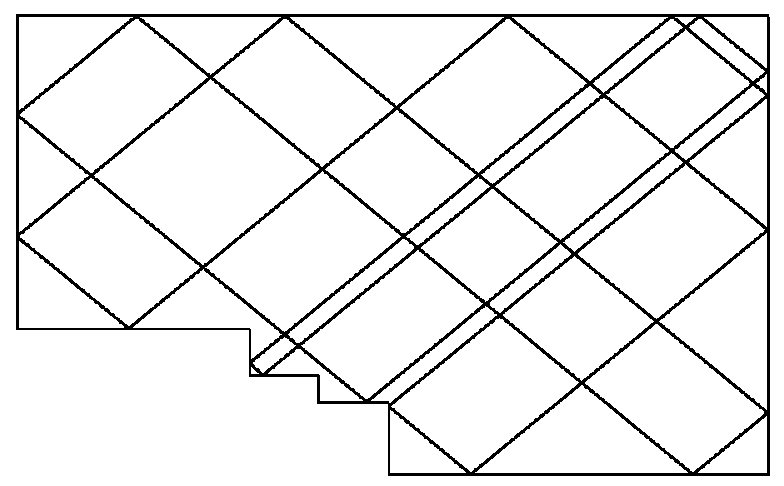

FIG. 2. An example of a periodic orbit in the 3-step billiard. Note that adjacent parallel rays are also periodic so that a family of periodic orbits exists. Its extent is limited by the $3 \pi / 2$ vertices. 


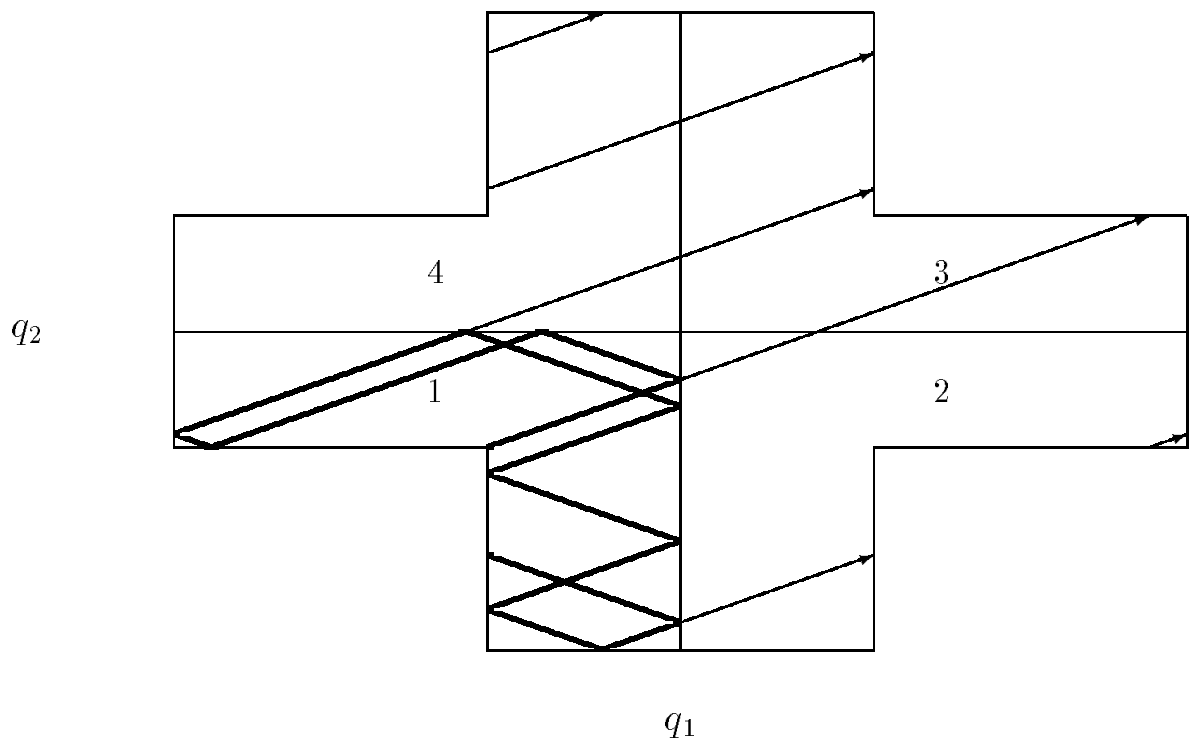

FIG. 3. The singly connected region for an L-shaped billiard consists of four copies with edges appropriately identified. A trajectory originating near the $3 \pi / 2$ vertex in 1 is plotted in configuration space using bold lines and the corresponding unfolded trajectory is also shown. The latter consists of parallel segments and the trajectory can be parametrized by the angle $\varphi$ that it makes for example with the $q_{1}$ axis. 


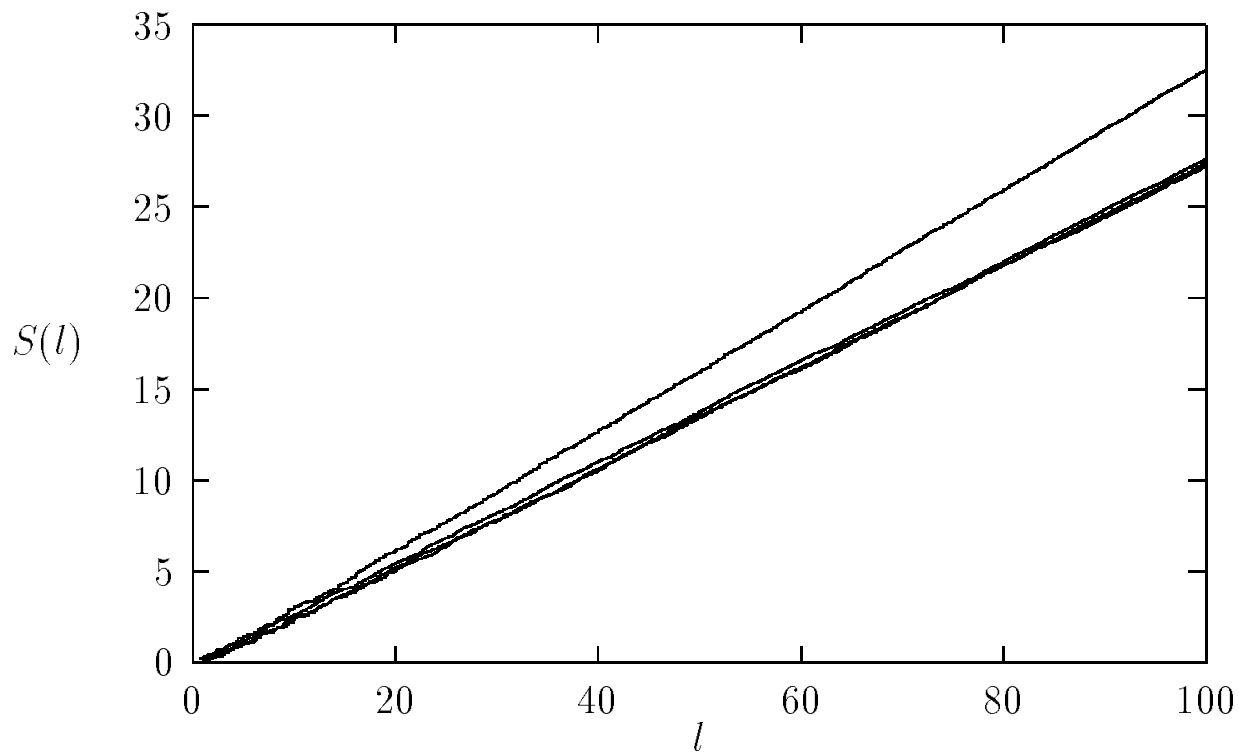

FIG. 4. A demonstration of the sum rule for the 1-step billiard. The curve with slope significantly different corresponds to a degenerate example. 


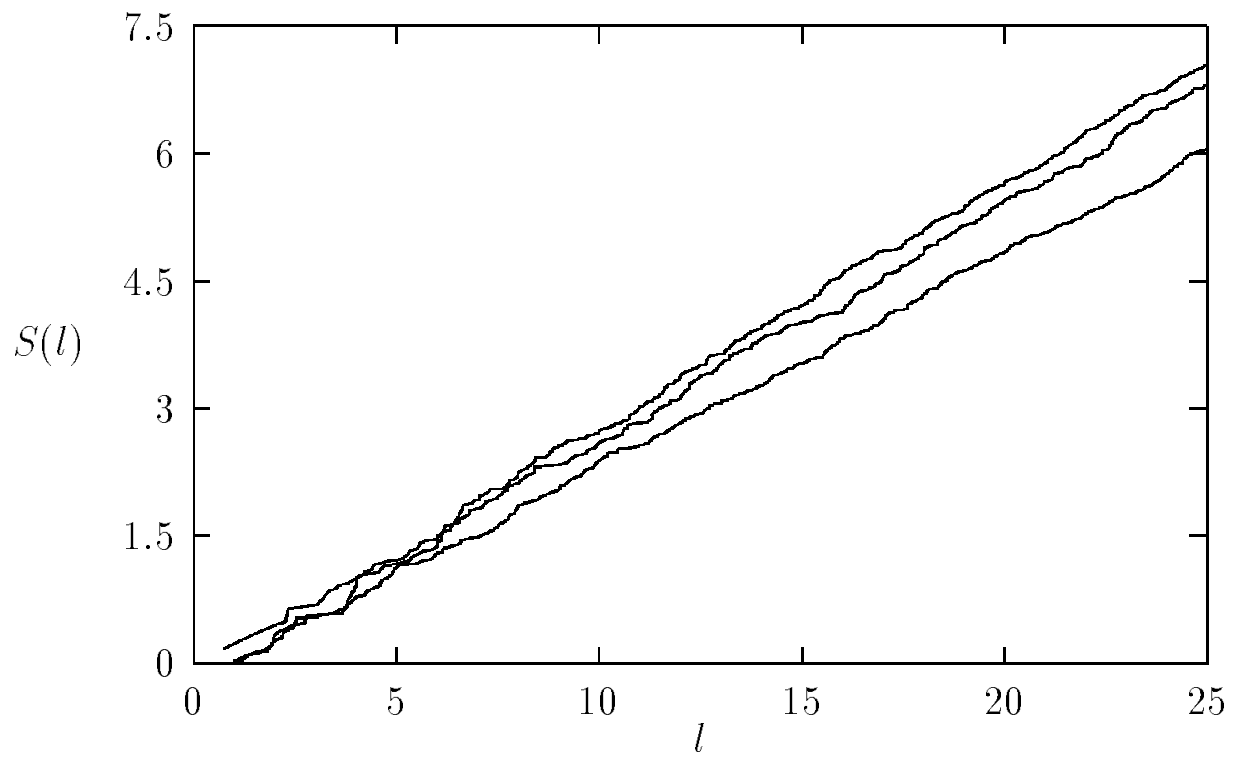

FIG. 5. The sum rule for a 1,2 and 3-step billiard. They are respectively the middle,bottom and top curves. 


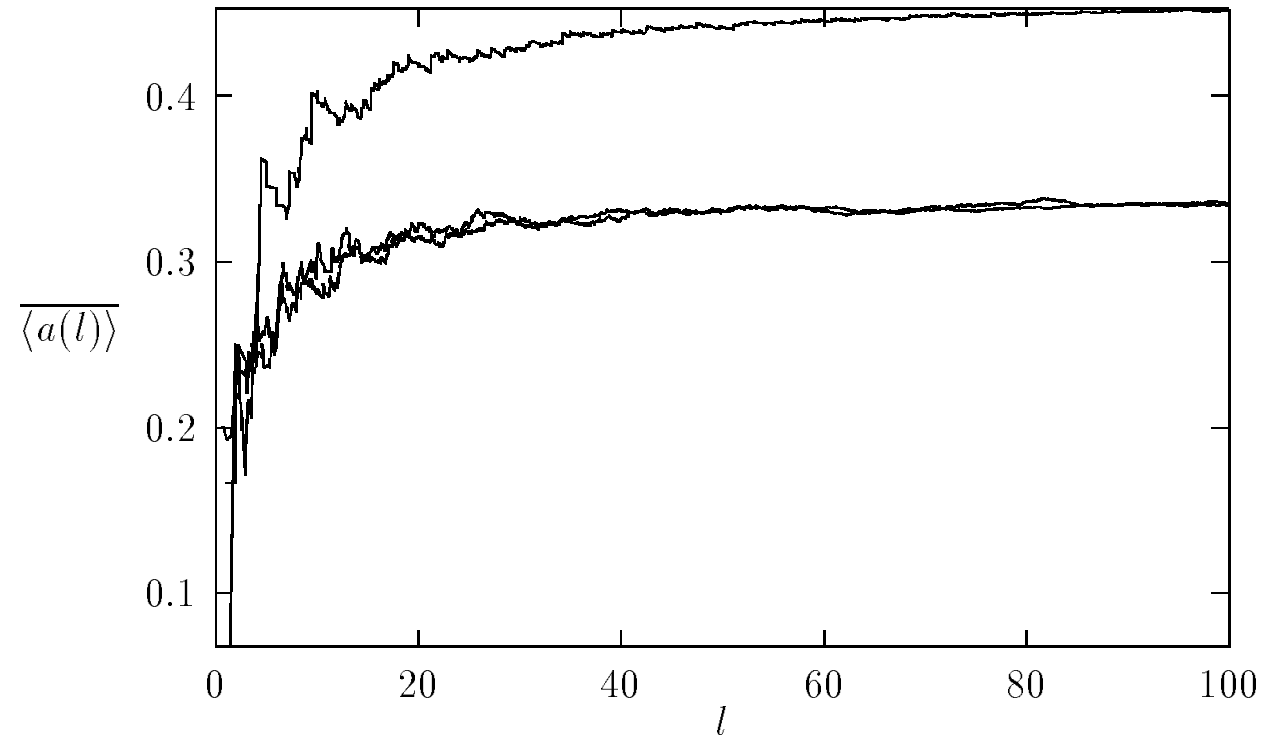

FIG. 6. The normalized average area, $\overline{\langle a(l)\rangle}=\langle a(l)\rangle / N A$ as a function of the length $l$ for 3 examples of the 1-step billiard. The saturation value differs for the degenerate example mentioned earlier 


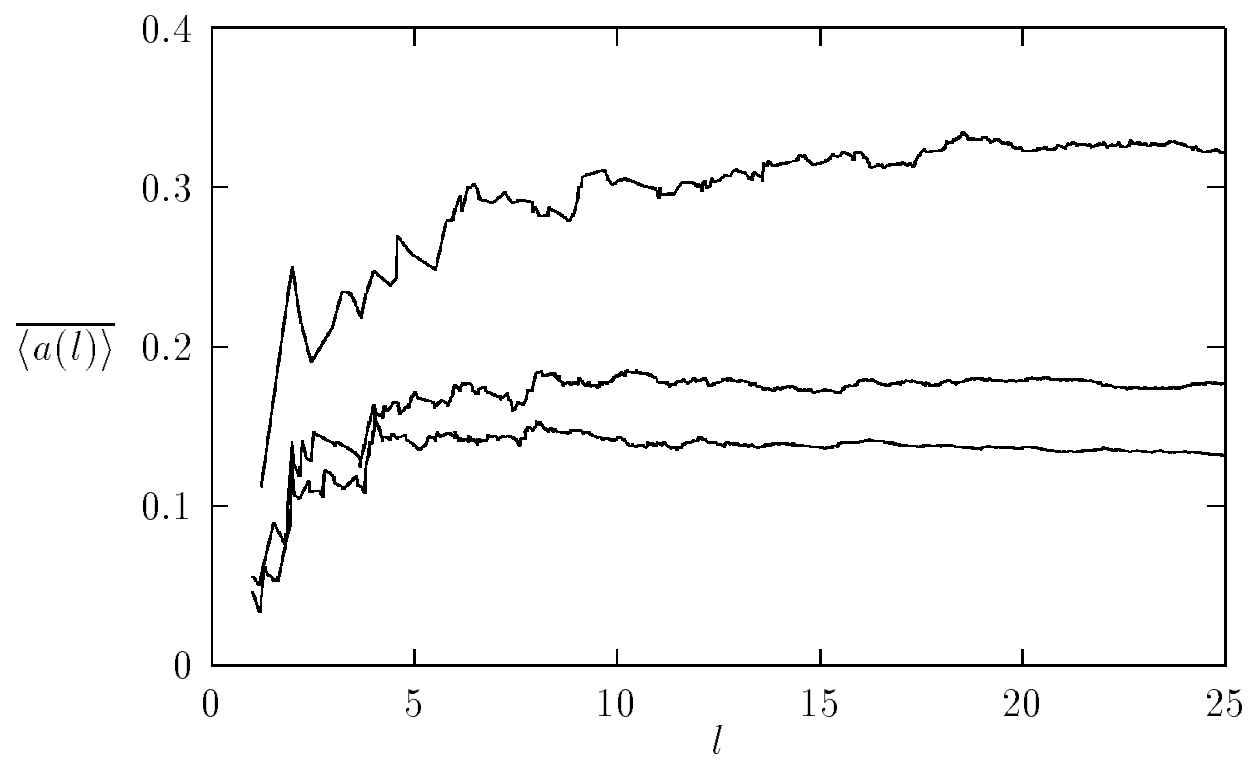

FIG. 7. The normalized area for an example each of the 1-step (top), 2-step (middle) and 3-step (bottom) billiards. 


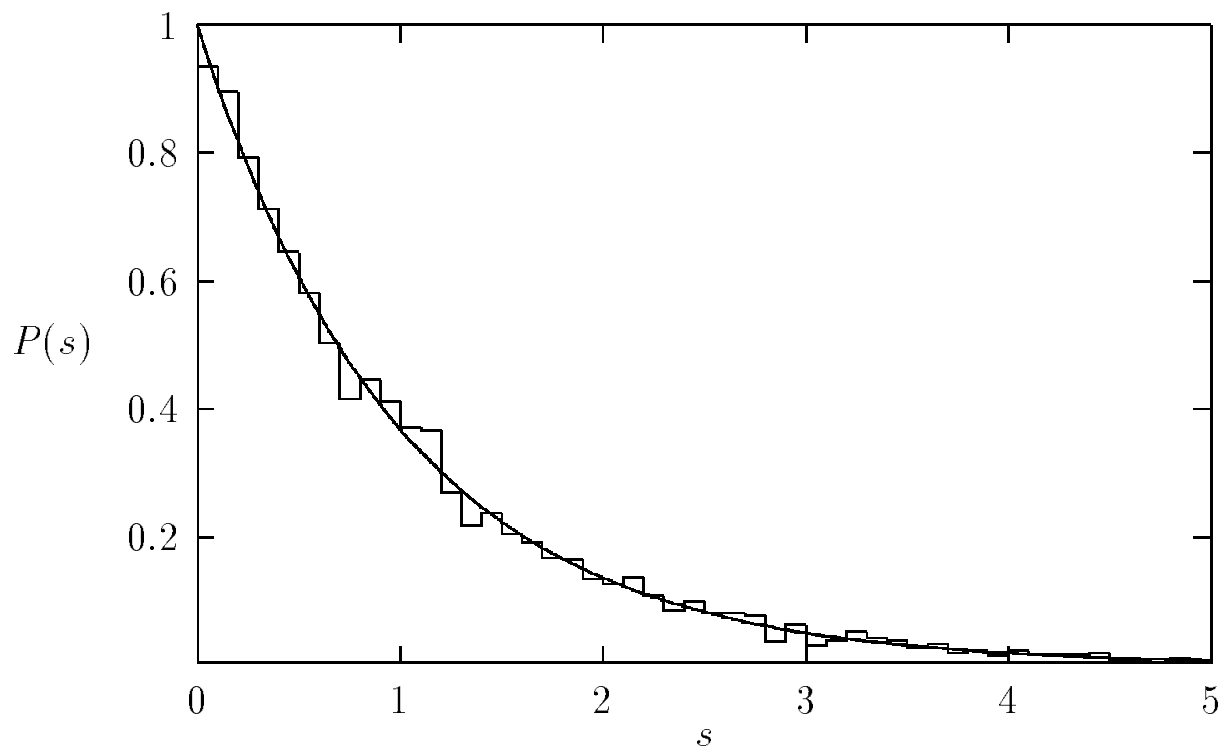

FIG. 8. The nearest neighbour spacings distribution for a non-degenerate example of a 1-step billiard. Also plotted is the Poisson distribution. 


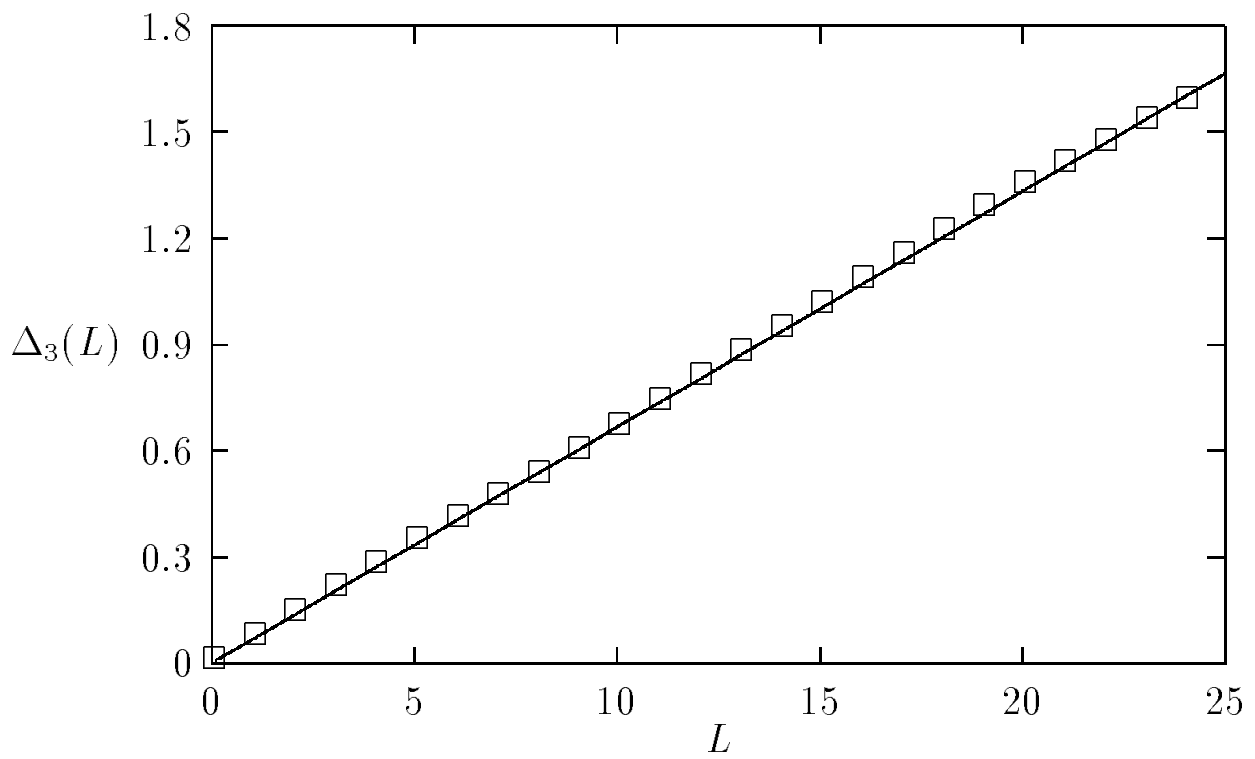

FIG. 9. The spectral rigidity for the 1-step billiard together with the $L / 15$ line. 


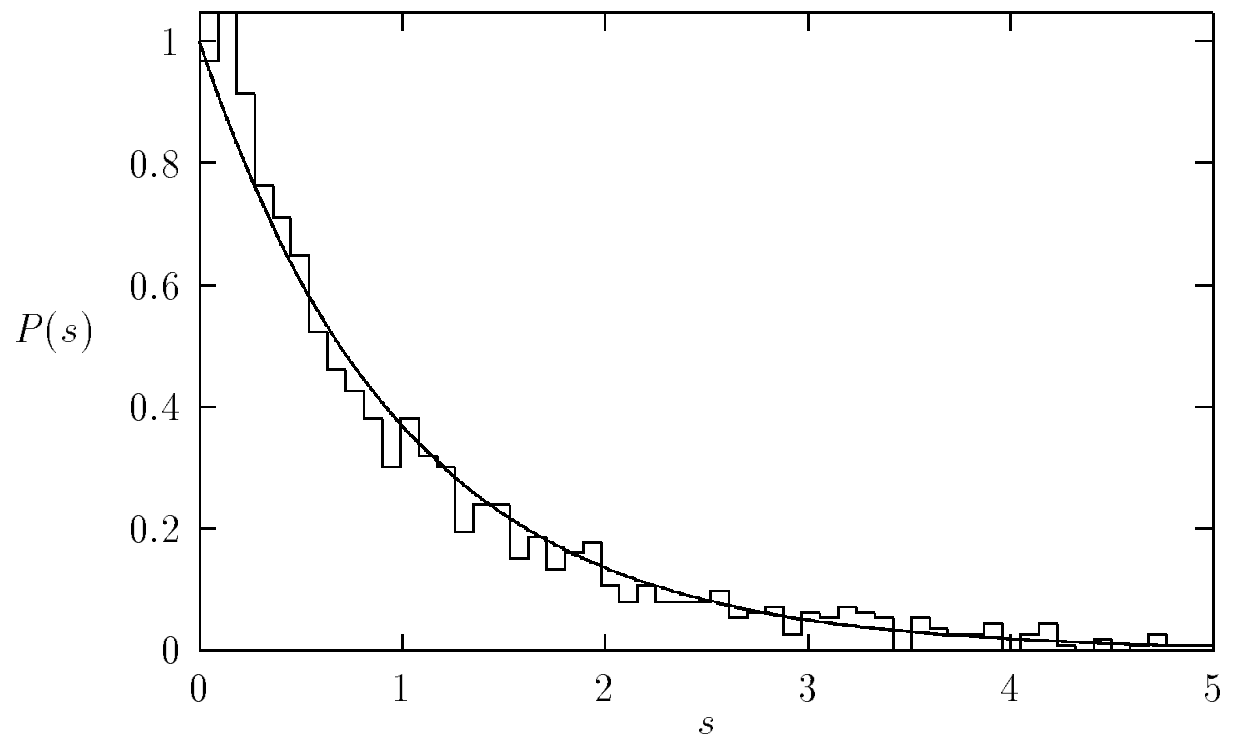

FIG. 10. The nearest neighbour distribution for a 3-step billiard. The available lengths are much smaller and minor deviations from Poisson behaviour can be observed 


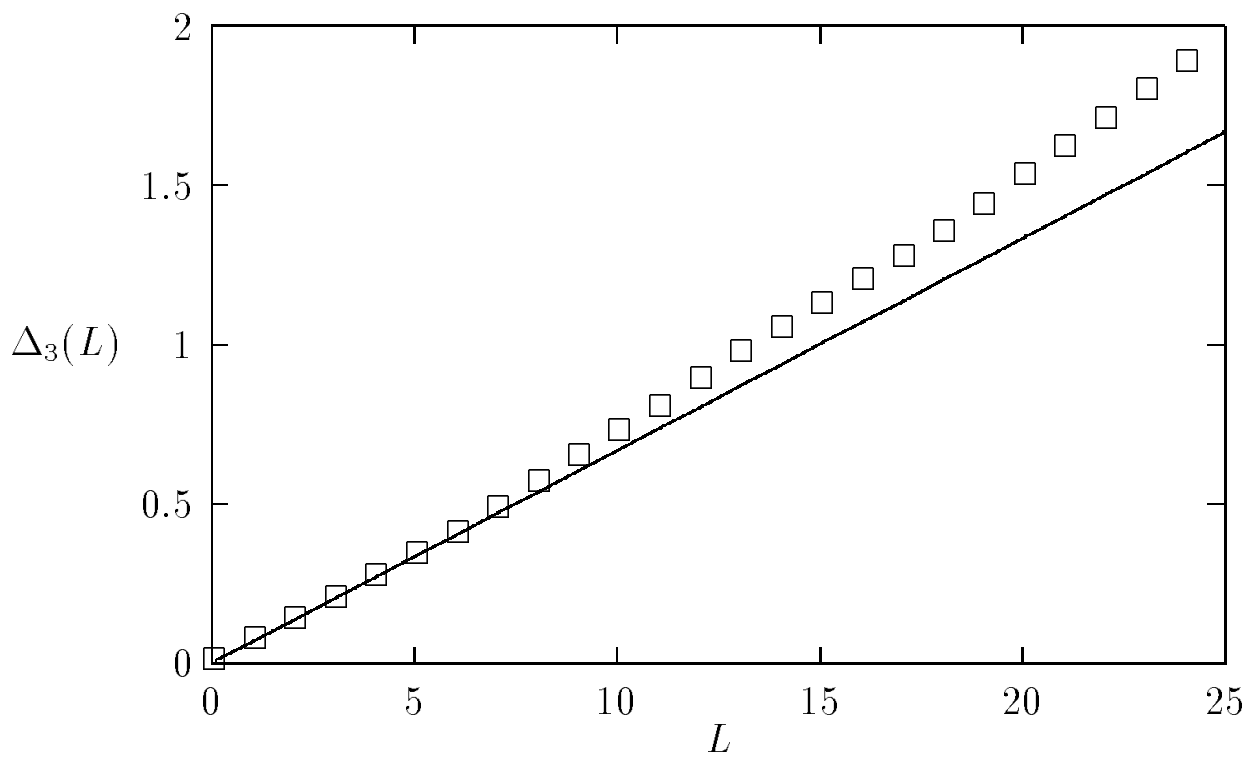

FIG. 11. The spectral rigidity for the 3-step billiard considered earlier. The deviations from Poisson behaviour is more pronounced for this measure. 